Anal. Theory Appl.

Vol. 28, No. 1 (2012), 58-64

\title{
A REMARK ON CERTAIN DIFFERENTIAL INEQUALITIES INVOLVING $p$-VALENT FUNCTIONS
}

\author{
Sukhwinder Singh Billing \\ (Baba Banda Singh Bahadur Engineering College, India)
}

Received Aug. 19, 2011

\begin{abstract}
In the present paper, we study certain differential inequalities involving $p$-valent functions and obtain sufficient conditions for uniformly $p$-valent starlikeness and uniformly $p$-valent convexity. We also offer a correct version of some known criteria for uniformly $p$-valent starlike and uniformly $p$-valent convex functions.
\end{abstract}

Key words: $p$-valent function, uniformly starlike function, uniformly convex function, uniformly close-to-convex function

AMS (2010) subject classification: 30C $80,30 \mathrm{C} 45$

\section{Introduction}

Let $\mathcal{A}_{p}$ denote the class of functions of the form

$$
f(z)=z^{p}+\sum_{k=p+1}^{\infty} a_{k} z^{k}, \quad p \in \mathbf{N}=\{1,2,3, \cdots\},
$$

which are analytic and $p$-valent in the open unit disk $\mathbf{E}=\{z \in \mathbf{C}:|z|<1\}$. A function $f \in \mathcal{A}_{p}$ is said to be uniformly $p$-valent starlike in $\mathbf{E}$ if

$$
\Re\left(\frac{z f^{\prime}(z)}{f(z)}\right)>\left|\frac{z f^{\prime}(z)}{f(z)}-p\right|, \quad z \in \mathbf{E} .
$$

We denote by $U S_{p}^{*}$, the class of uniformly $p$-valent starlike functions. A function $f \in \mathcal{A}_{p}$ is said to be uniformly $p$-valent convex in $\mathbf{E}$ if

$$
\Re\left(1+\frac{z f^{\prime \prime}(z)}{f^{\prime}(z)}\right)>\left|1+\frac{z f^{\prime \prime}(z)}{f^{\prime}(z)}-p\right|, \quad z \in \mathbf{E} .
$$

Let $U C_{p}$ denote the class of uniformly $p$-valent convex functions. A function $f \in \mathcal{A}_{p}$ is said to be uniformly $p$-valent close-to-convex in $\mathbf{E}$ if

$$
\Re\left(\frac{z f^{\prime}(z)}{g(z)}\right)>\left|\frac{z f^{\prime}(z)}{g(z)}-p\right|, \quad z \in \mathbf{E},
$$


for some $g \in U S_{p}^{*}$. Let $U C C_{p}$ denote the class of all such functions. Note that the function $g(z) \equiv$ $z^{p} \in \mathcal{A}_{p}$ and satisfies the condition (1.1). Therefore, when we select $g(z) \equiv z^{p}$, in condition (1.2), it reduces to

$$
\Re\left(\frac{f^{\prime}(z)}{z^{p-1}}\right)>\left|\frac{f^{\prime}(z)}{z^{p-1}}-p\right|, \quad z \in \mathbf{E} .
$$

Hence, a function $f \in \mathcal{A}_{p}$ is uniformly $p$-valent close-to-convex in $\mathbf{E}$ if it satisfies the condition (1.3).

In 1991, Goodman ${ }^{[2]}$ introduced the concept of uniformly starlike and uniformly convex functions. He defined uniformly starlike and uniformly convex functions as functions $f \in \mathcal{A}$ with the geometric property that the image of every circular arc contained in $\mathbf{E}$, with center $\zeta \in \mathbf{E}$, is starlike with respect to $f(\zeta)$ and convex respectively.

In 1993 Ronning ${ }^{[4]}$ studied the class of uniformly convex functions and obtained an interesting criterion for $f \in \mathcal{A}$ to be uniformly convex in $\mathbf{E}$. He proved that a function $f \in \mathcal{A}$ is uniformly convex if and only if

$$
\Re\left(1+\frac{z f^{\prime \prime}(z)}{f^{\prime}(z)}\right)>\left|\frac{z f^{\prime \prime}(z)}{f^{\prime}(z)}\right|, \quad z \in \mathbf{E} .
$$

For analytic function $f$ and analytic univalent function $g$ in $\mathbf{E}$, we say that $f$ is subordinate to $g$ in $\mathbf{E}$ and write as $f \prec g$ if $f(0)=g(0)$ and $f(\mathbf{E}) \subset g(\mathbf{E})$.

Let $\Phi: \mathbb{C}^{2} \times \mathbb{E} \rightarrow \mathbb{C}$ be an analytic function, $p$ an analytic function in $\mathbb{E}$ with $\left(p(z), z p^{\prime}(z) ; z\right) \in \mathbb{C}^{2} \times \mathbb{E}$ for all $z \in \mathbb{E}$ and $h$ be univalent in $\mathbb{E}$. Then the function $p$ is said to satisfy first order differential subordination if

$$
\Phi\left(p(z), z p^{\prime}(z) ; z\right) \prec h(z), \Phi(p(0), 0 ; 0)=h(0) .
$$

A univalent function $q$ is called a dominant of the differential subordination (1.4) if $p(0)=q(0)$ and $p \prec q$ for all $p$ satisfying (1.4). A dominant $\tilde{q}$ that satisfies $\tilde{q} \prec q$ for all dominants $q$ of (1.4) is said to be the best dominant of (1.4).

Define the parabolic domain $\Omega$ and the circular domain $O$ as follows:

$$
\Omega=\left\{u+i v: u>\sqrt{(u-p)^{2}+v^{2}}\right\}
$$

and

$$
O=\left\{u+i v: \sqrt{(u-p)^{2}+v^{2}}<\frac{p}{2}\right\} .
$$

Obviously $O \subset \Omega$. In 2008, Al-Kharsani and Al-Hajiry ${ }^{[1]}$ proved the following results for uniformly $p$-valent starlikeness and convexity.

Theorem 1.1. Let $f \in \mathcal{A}_{p}$ satisfy the following inequality

$$
\Re\left(\frac{1+\frac{z f^{\prime \prime}(z)}{f^{\prime}(z)}-p}{\frac{z f^{\prime}(z)}{f(z)}-p}\right)<1+\frac{2}{3 p},
$$

then $f$ is uniformly $p$-valent starlike in $\mathbb{E}$. 
Theorem 1.2. If $f \in \mathcal{A}_{p}$ satisfies the following inequality

$$
\Re\left(\frac{1+\frac{z f^{\prime \prime \prime}(z)}{f^{\prime \prime}(z)}-p}{1+\frac{z f^{\prime \prime}(z)}{f^{\prime}(z)}-p}\right)<1+\frac{2}{3 p-2},
$$

then $f$ is uniformly $p$-valent convex in $\mathbb{E}$.

We notice that the problem of normalization in inequality (1.5) and (1.6) occurs at $z=0$. For $f \in \mathcal{A}_{p}, \frac{z f^{\prime}(z)}{f(z)}=p, \frac{z f^{\prime \prime}(z)}{f^{\prime}(z)}=p-1$ and $\frac{z f^{\prime \prime \prime}(z)}{f^{\prime \prime}(z)}=p-2$ at $z=0$. Thus the left hand side of the inequality (1.5) takes indeterminate value i.e. $0 / 0$ and that of the inequality (1.6) takes value infinity at $z=0$. Hence it creates a problem for normalization in the open unit disk $\mathbb{E}$. Noticing the above problem in case of results of Al-Kharsani and Al-Hajiry ${ }^{[1]}$, we establish here certain criteria for uniformly $p$-valent starlikeness and uniformly $p$-valent convexity of the members of the class $\mathcal{A}_{p}$ which also present a correct version of above results. To prove our results, we shall use Jack's lemma stated below.

Lemma 1.1. ${ }^{[3]}$ Suppose $w$ is a nonconstant analytic function in $\mathbb{E}$ with $w(0)=0$. If $|w(z)|$ attains its maximum value at a point $z_{0} \in \mathbb{E}$ on the circle $|z|=r<1$, then

$$
z_{0} w^{\prime}\left(z_{0}\right)=m w\left(z_{0}\right)
$$

where $m \geq 1$, is some real number.

\section{Main Results}

Theorem 2.1. Let $f \in \mathcal{A}_{p}$ satisfy the differential inequality

$$
\left|\frac{1+\frac{z f^{\prime \prime}(z)}{f^{\prime}(z)}}{\frac{z f^{\prime}(z)}{f(z)}}-1\right|<\frac{2}{9 p}, z \in \mathbb{E},
$$

then $f \in U S_{p}^{*}$.

Proof. Let us write

$$
\frac{z f^{\prime}(z)}{f(z)}-p=\frac{p}{2} w(z)
$$

where $w$ is analytic in $\mathbb{E}$ with $w(0)=0$. Now we will show that

$$
|w(z)|<1, z \in \mathbb{E} .
$$

If $|w(z)| \nless 1$, by Lemma 1.1, there exists $z_{0},\left|z_{0}\right|<1$ such that $\left|w\left(z_{0}\right)\right|=1$ and $z_{0} w^{\prime}\left(z_{0}\right)=k w\left(z_{0}\right)$ where $k \geq 1$. When we put

$$
w\left(z_{0}\right)=e^{i \theta}
$$


we have

$$
\begin{aligned}
\left|\frac{1+\frac{z_{0} f^{\prime \prime}\left(z_{0}\right)}{f^{\prime}\left(z_{0}\right)}}{\frac{z_{0} f^{\prime}\left(z_{0}\right)}{f\left(z_{0}\right)}}-1\right| & =\left|\frac{2}{p} \frac{z_{0} w^{\prime}\left(z_{0}\right)}{\left(2+w\left(z_{0}\right)\right)^{2}}\right| \\
& =\frac{2}{p}\left|\frac{k w\left(z_{0}\right)}{\left(2+w\left(z_{0}\right)\right)^{2}}\right| \\
& =\frac{2}{p}\left|\frac{k e^{i \theta}}{\left(2+e^{i \theta}\right)^{2}}\right| \\
& \geq \frac{2}{p} \frac{1}{\left(2+e^{i \theta}\right)^{2}} \geq \frac{2}{9 p},
\end{aligned}
$$

which is a contradiction to (2.1). Therefore, we must have

$$
|w(z)|<1, z \in \mathbb{E}
$$

and so we have

$$
\left|\frac{z f^{\prime}(z)}{f(z)}-p\right|<\frac{p}{2}
$$

which shows that $\frac{z f^{\prime}(z)}{f(z)}$ lies in a circular domain $O$ for all $z \in \mathbb{E}$. Therefore, $\frac{z f^{\prime}(z)}{f(z)}$ takes values in the parabolic domain $\Omega$ for all $z$ in $\mathbb{E}$ and hence

$$
\Re\left(\frac{z f^{\prime}(z)}{f(z)}\right)>\left|\frac{z f^{\prime}(z)}{f(z)}-p\right|, z \in \mathbb{E}
$$

which completes the proof.

Theorem 2.2. If $f \in \mathcal{A}_{p}$ satisfies the differential inequality

$$
\left|\frac{2+\frac{z f^{\prime \prime \prime}(z)}{f^{\prime \prime}(z)}}{1+\frac{z f^{\prime \prime}(z)}{f^{\prime}(z)}}-1\right|<\frac{2}{3(3 p-2)}, z \in \mathbb{E},
$$

then $f \in U C_{p}$.

Proof. Let us write

$$
1+\frac{z f^{\prime \prime}(z)}{f^{\prime}(z)}-p=\frac{p}{2} w(z)
$$

where $w$ is analytic in $\mathbb{E}$ with $w(0)=0$. To show that

$$
|w(z)|<1, z \in \mathbb{E} .
$$

We suppose that $|w(z)| \nless 1$, therefore in view of Lemma 1.1, there exists $z_{0},\left|z_{0}\right|<1$ such that $\left|w\left(z_{0}\right)\right|=1$ and

$$
z_{0} w^{\prime}\left(z_{0}\right)=k w\left(z_{0}\right)
$$


where $k \geq 1$. On writing $w\left(z_{0}\right)=e^{i \theta}$, we obtain

$$
\begin{aligned}
\left|\frac{2+\frac{z_{0} f^{\prime \prime \prime}\left(z_{0}\right)}{f^{\prime \prime}\left(z_{0}\right)}}{1+\frac{z_{0} f^{\prime \prime}\left(z_{0}\right)}{f^{\prime}\left(z_{0}\right)}}-1\right| & =\left|\frac{z_{0} w^{\prime}\left(z_{0}\right)}{\left(2+w\left(z_{0}\right)\right)\left(\frac{p}{2}\left(2+w\left(z_{0}\right)\right)-1\right)}\right| \\
& =\left|\frac{k w\left(z_{0}\right)}{\left(2+w\left(z_{0}\right)\right)\left(\frac{p}{2}\left(2+w\left(z_{0}\right)\right)-1\right)}\right| \\
& =\left|\frac{k e^{i \theta}}{\left(2+e^{i \theta}\right)\left(\frac{p}{2}\left(2+e^{i \theta}\right)-1\right)}\right| \\
& =\frac{k}{\left|\left(2+e^{i \theta}\right)\left(\frac{p}{2}\left(2+e^{i \theta}\right)-1\right)\right|} \\
& \geq \frac{2}{3(3 p-2)},
\end{aligned}
$$

which is a contradiction to (2.2). Therefore, we must have

$$
|w(z)|<1, z \in \mathbb{E}
$$

and thus we get

$$
\left|1+\frac{z f^{\prime \prime}(z)}{f^{\prime}(z)}-p\right|<\frac{p}{2}
$$

which shows that $1+\frac{z f^{\prime \prime}(z)}{f^{\prime}(z)}$ lies in the circular domain $O$. Therefore, for all $z$ in $\mathbb{E}, 1+\frac{z f^{\prime \prime}(z)}{f^{\prime}(z)}$ takes values in the parabolic domain $\Omega$ and hence

$$
\Re\left(1+\frac{z f^{\prime \prime}(z)}{f^{\prime}(z)}\right)>\left|1+\frac{z f^{\prime \prime}(z)}{f^{\prime}(z)}-p\right|,
$$

which completes the proof.

Theorem 2.3. If $f \in \mathcal{A}_{p}$ satisfies the differential inequality

$$
\left|1+\frac{z f^{\prime \prime}(z)}{f^{\prime}(z)}-p\right|<\frac{1}{3}, z \in \mathbb{E},
$$

then $f \in U C C_{p}$.

Proof. Let us write

$$
\frac{f^{\prime}(z)}{z^{p-1}}-p=\frac{p}{2} w(z),
$$

where $w$ is analytic in $\mathbb{E}$ with $w(0)=0$. We show that

$$
|w(z)|<1, z \in \mathbb{E} .
$$

If we suppose $|w(z)| \nless 1$, then by Lemma 1.1, there exists $z_{0},\left|z_{0}\right|<1$ such that $\left|w\left(z_{0}\right)\right|=1$ and

$$
z_{0} w^{\prime}\left(z_{0}\right)=k w\left(z_{0}\right)
$$


where $k \geq 1$. Writing $w\left(z_{0}\right)=e^{i \theta}$, we have

$$
\begin{aligned}
\left|1+\frac{z_{0} f^{\prime \prime}\left(z_{0}\right)}{f^{\prime}\left(z_{0}\right)}-p\right| & =\left|\frac{z_{0} w^{\prime}\left(z_{0}\right)}{2+w\left(z_{0}\right)}\right| \\
& =\left|\frac{k w\left(z_{0}\right)}{2+w\left(z_{0}\right)}\right| \\
& =\left|\frac{k e^{i \theta}}{2+e^{i \theta}}\right| \\
& \geq \frac{1}{2+e^{i \theta}} \geq \frac{1}{3},
\end{aligned}
$$

which is a contradiction to (2.3). Therefore, we must have $|w(z)|<1, z \in \mathbb{E}$ and thus

$$
\left|\frac{f^{\prime}(z)}{z^{p-1}}-p\right|<\frac{p}{2}
$$

which shows that $\frac{f^{\prime}(z)}{z^{p-1}}$ lies in a circular domain $O$ for all $z \in \mathbb{E}$. Therefore, $\frac{f^{\prime}(z)}{z^{p-1}}$ takes values in the parabolic domain $\Omega$ for all $z$ in $\mathbb{E}$ and hence

$$
\Re\left(\frac{f^{\prime}(z)}{z^{p-1}}\right)>\left|\frac{f^{\prime}(z)}{z^{p-1}}-p\right|, z \in \mathbb{E},
$$

which completes the proof.

\section{Deductions}

Using the concept of subordination, we put the above studied differential inequalities in differential subordination form and then we derive the results in the form of differential inequalities involving real part only which are then compared with the results of Al-Kharsani and Al-Hajiry ${ }^{[3]}$. In view of Theorem 2.1 and Theorem 2.2, we obtain the next two results respectively.

Corollary 3.1. For $z$ in $\mathbb{E}$, if $f \in \mathcal{A}_{p}$ satisfies

$$
\frac{1+\frac{z f^{\prime \prime}(z)}{f^{\prime}(z)}}{\frac{z f^{\prime}(z)}{f(z)}} \prec 1+\frac{2}{9 p} z,
$$

or

$$
\Re\left(\frac{1+\frac{z f^{\prime \prime}(z)}{f^{\prime}(z)}}{\frac{z f^{\prime}(z)}{f(z)}}\right)<1+\frac{2}{9 p},
$$

then $f \in U S_{p}^{*}$. 
64 S. S. Billing : A Remark on Certain Differential Inequalities Involving p-valent Functions

Corollary 3.2. Let $f \in \mathcal{A}_{p}$ satisfy

$$
\frac{2+\frac{z f^{\prime \prime \prime}(z)}{f^{\prime \prime}(z)}}{1+\frac{z f^{\prime \prime}(z)}{f^{\prime}(z)}} \prec 1+\frac{2}{3(3 p-2)} z, z \in \mathbb{E},
$$

or

$$
\Re\left(\frac{2+\frac{z f^{\prime \prime \prime}(z)}{f^{\prime \prime}(z)}}{1+\frac{z f^{\prime \prime}(z)}{f^{\prime}(z)}}\right)<1+\frac{2}{3(3 p-2)},
$$

then $f \in U C_{p}$.

Remark 3.1. The inequality (3.1) presents a correct version of the result of Al-Kharsani and Al-Hajiry [3] stated in Theorem 1.1 whereas the inequality (3.2) gives the same for the result in Theorem 1.2.

From Theorem 2.3, we deduce:

Corollary 3.3. Let $f \in \mathcal{A}_{p}$ satisfy

$$
1+\frac{z f^{\prime \prime}(z)}{f^{\prime}(z)} \prec p+\frac{1}{3} z, z \in \mathbb{E},
$$

then $f \in U C C_{p}$.

Now Corollary 3.3, in turn, gives the following result of Al-Kharsani and Al-Hajiry ${ }^{[3]}$.

Corollary 3.4. If $f \in \mathcal{A}_{p}$ satisfies the following inequality

$$
\Re\left(\frac{z f^{\prime \prime}(z)}{f^{\prime}(z)}\right)<p-\frac{2}{3},
$$

then $f$ is uniformly p-valent close-to-convex in $\mathbb{E}$.

\section{References}

[1] Al-Kharsani, H. A. and Al-Hajiry, S. S., A Note on Certain Inequalities for $p$-valent Functions, J. Ineq. Pure and Appl. Math., 9:3(2008), Article 90, 1-6.

[2] Goodman, A. W., On Uniformly Starlike Functions, J. Math. Anal. Appl., 155(1991), 364-370.

[3] Jack, I. S., Functions Starlike and Convex of Order $\alpha$, J. London Math. Soc., 3(1971), 469-474.

[4] Ronning, F., Uniformly Convex Functions and a Corresponding Class of Starlike Functions, Proc. Amer. Math. Soc., 118(1993), 189-196.

Deaprtment of Applied Sciences

Baba Banda Singh Bahadur Engineering College

Fatehgarh Sahib-140 407 (Punjab)

India

E-mail: ssbilling@gmail.com 